\title{
Biomarkers inform clinical trials and pathogenesis in systemic sclerosis
}

\author{
R Lafyatis \\ From 5th European Workshop on Immune-Mediated Inflammatory Diseases \\ Sitges-Barcelona, Spain. 1-3 December 2010
}

\section{Introduction}

Biomarkers of disease are becoming increasingly recognized as central in design of clinical trials. Biomarkers allow us to link what "might be" as defined by in vitro and animal model studies with what "is" happening in disease pathogenesis. In scleroderma or systemic sclerosis (SSc) this is particularly important for implementing new strategies for clinical trials and understanding pathogenesis.

\section{Aim}

We investigated blood and skin from SSc patients utilizing microarray and proteomic approaches. Using the results of these studies we evaluated existing and created new animal models of SSc.

\section{Patients and methods}

Patients with diffuse cutaneous (dSSc), having typically extensive skin fibrosis and limited cutaneous SSc (ISSc), having less extensive skin fibrosis but occasionally suffering from the deadly complication of pulmonary arterial hypertension (PAH) were recruited for cross-sectional and longitudinal studies of gene expression and cytokine levels. Identified biomarkers were correlated with clinical data. New murine models were developed by delivery of putative SSc mediators by subcutaneous osmotic pump.

\section{Results}

Skin from patients with dSSc showed upregulated expression of TGFß- and IFN-regulated genes, permitting development of a four-gene biomarker of skin disease correlating highly with the modified Rodnan skin score, the current standard for measuring the extent of skin involvement [1]. Peripheral blood mononuclear cell

Dept. of Medicine, Rheumatology Section, Boston University Medical Center, Boston, MA, USA
mRNA expression and sera from 1 SSc patients with PAH showed upregulated markers of monocyte activation: IFNGR1, IL13a1 and CCR1; and circulating markers of innate immunity: IL- 6 and TNF-a; and vascular activation/injury: vWF, VCAM-1 and VEGF [2]. Continuous subcutaneous delivery of the toll-like receptor 3 ligand, poly(I:C), reproduces many of these features in mice, including upregulated IFN- and TGFb-regulated gene expression [3].

\section{Conclusions}

Identified biomarkers of SSc disease severity and complications provide strong rationale for trials of therapeutics targeting upregulated mediators. These biomarkers also provide potential surrogate outcome measures for clinical trials and insight into pathogenesis. Mouse models showing immune dysregulation including bleomycin induced skin disease, SSc-like graft-versushost-disease and chronic poly(I:C) stimulation place innate immunity central in SSc-associated fibrosis [4].

Published: 25 November 2010

\section{References}

1. Farina G, Lafyatis D, Lemaire R, Lafyatis R: A four-gene biomarker predicts skin disease in patients with diffuse cutaneous systemic sclerosis. Arthritis Rheum 2010, 62:580-588.

2. Pendergrass SA, Hayes E, Farina G, Lemaire R, Farber HW, Whitfield ML, Lafyatis R: Limited systemic sclerosis patients with pulmonary arterial hypertension show biomarkers of inflammation and vascular injury. PLOS One 2010, 5.

3. Farina GA, York MR, Di Marzio M, Collins CA, Meller S, Homey B, Rifkin IR, Marshak-Rothstein A, Radstake TR, Lafyatis R: Poly(I:C) Drives Type I IFNand TGFbeta-Mediated Inflammation and Dermal Fibrosis Simulating Altered Gene Expression in Systemic Sclerosis. J Invest Dermatol 2010.

4. Lafyatis $R$, York M: Innate immunity and inflammation in systemic sclerosis. Curr Opin Rheumatol 2009, 21:617-622.

doi:10.1186/1479-5876-8-S1-I14

Cite this article as: Lafyatis: Biomarkers inform clinical trials and pathogenesis in systemic sclerosis. Journal of Translational Medicine 2010 8(Suppl 1):114. 